\title{
Natriuretic Peptides Find a New Home in Pulmonology
}

\author{
Thenral Socrates Christian Müller \\ Department of Internal Medicine, University Hospital Basel, Basel, Switzerland
}

Diagnosing the early signs of heart failure, although seemingly clear cut, still remains elusive in the scenario of acute decompensation at the level of the primary care physician. This is especially true in those patients with coexisting respiratory disorders such as chronic obstructive pulmonary disease who present with dyspnea, 'cardiac asthma', or have a long history of smoking $[1,2]$. A vicious cycle of respiratory and right and left cardiac disease states influencing each other exists, where dyspnea is the common presenting symptom. A rapid screening method to stratify and differentiate dyspnea of cardiac origin from that of a pulmonary origin is imperative for both the patient and the physician [3].

Biomarkers are fast becoming a reliable and widely used approach to determine the extent of heart failure [4]. Natriuretic peptides, such as brain natriuretic peptide (BNP) and N-terminal fragment (NT)-proBNP are endogenous peptides rapidly synthesized and secreted by the atrial and ventricular cardiomyocytes in response to myocardial stretch, raised left ventricular end diastolic pressure and left ventricular dysfunction $[1,2]$. BNP is an active hormone causing natriuresis, inhibition of the renin aldosterone system, vasorelaxation, and antifibrotic, antihypertrophic and lusitropic effects. NT-proBNP does not seem to have a biological effect. BNP gene expression is upregulated in disease states such as congestive heart failure. As there are no storage granules for these pep- tides, increased secretion occurs specifically after mRNA over expression [5]. Both markers are raised in systolic and diastolic heart failure and correlate to echocardiographic findings [2].

In this issue of Respiration, Wieshammer et al. [6] describe the utility of NT-proBNP for pulmonologists determining if an underlying cardiac etiology exists in patients referred with dyspnea. In this prospective monocenter study conducted in the pulmonology service of an academic teaching hospital, over a 2 year period, the evaluation of dyspnea of at least 2 weeks duration was performed using NT-proBNP as an index test. Patients underwent usual diagnostic testing for heart and lung disease, and as the examining physician was blinded to the index test, treatment was not changed based on NT-pro-BNP levels. In this study, 738 patients were enrolled, 41 were excluded due to missing data and therefore data from 697 patients were available for final analysis. Cardiological findings were categorized into 6 groups to help in the statistical evaluation of the enrolled patients. The groups were as follows. Group 1: left ventricular hypertrophy. Group 2: exercise induced myocardial ischemia. Group 3: at least mild aortic stenosis, at least moderate mitral regurgitation, severe tricuspid regurgitation without evidence of pulmonary hypertension, atrial septal defect or pericardial effusion. Group 4: pulmonary hypertension. Group 5: atrial fibrillation or left bundle branch block. Group 6:

\section{KARGER}

Fax +4161306 1234 E-Mail karger@karger.ch www.karger.com (c) 2009 S. Karger AG, Basel

0025-7931/09/0774-0368\$26.00/0

Accessible online at:

www.karger.com/res
Prof. Dr. Christian Müller

Department of Internal Medicine, University Hospital Basel

Petersgraben 4

$\mathrm{CH}-4031$ Basel (Switzerland)

Tel. +41 6132865 49, Fax +41 6126553 53, E-Mail chmueller@uhbs.ch 
impaired left ventricular systolic function. NT-proBNP was found to be elevated in each of the 6 different cardiological diagnoses compared to patients with no cardiological findings. Sole cardiac disease was found in 5.9\%, a combination of pulmonary and cardiac disease was found in $19.4 \%$, and sole respiratory findings were seen in $59.2 \%$. Furthermore, cardiac disease was found in $43.5 \%$ of patients with COPD and left ventricular systolic function was impaired in $8.1 \%$ of these patients.

For the 6 cardiological groups that were created in this study, of which all had elevated NT-proBNP levels, the following standardized regression coefficients were seen: Group 1: $\beta=0.33$, 95\% CI $0.03-0.62, p=0.03$; group 2: $\beta=0.73$, 95\% CI $0.11-1.35, p=0.02$; group $3: \beta=0.93$, 95\% CI 0.50-1.36, $\mathrm{p}<0.0001$; group $4: \beta=1.14,95 \% \mathrm{CI}$ $0.84-1.44, \mathrm{p}<0.0001$; group $5: \beta=1.22,95 \%$ CI $0.90-1.53$, $\mathrm{p}<0.0001$; group 6 : $\beta=1.94,95 \%$ CI $1.52-2.35, \mathrm{p}<0.0001$ [6].

This study therefore supports and validates current viewpoints that the measurement of natriuretic peptides is beneficial not only in the emergency department, but also in the primary care setting as an early diagnostic test in cases presenting with dyspnea without an evident cardiac etiology $[7,8]$. This finding is of great clinical relevance as the vast majority of previous studies on natriuretic peptides were conducted in the emergency department, while the majority of patients with dyspnea primarily present to doctors in private practice. The pulmonologist benefits from a relatively rapid stratification as to the cause of dyspnea. This study presents a novel approach in patient diagnosis by introducing natriuretic peptide measurements in a nonemergency setting. This brings to light the fact that natriuretic peptides can indicate patients with mild chronic dyspnea, thereby giving the primary care physician a window of opportunity to treat and prevent an episode of acute exacerbation. There- fore, natriuretic peptide assessment in this setting has the potential to be not just an extra tool for the physician but an indicator that jump starts diagnosis and treatment. This is further supported by recent findings from a prospective observational study that included consecutive patients with unexplained exercise intolerance referred for cardiopulmonary exercise testing. Elevated levels of BNP and NT-proBNP were found to be simple, rapid and accurate tools in the identification of cardiovascular limitation of exercise [9].

Wieshammer et al. [6] proposed a new use for cardiac biomarkers in the primary care setting. The practicality of this in terms of obtaining rapid lab results and then adjusting patient therapy is unknown. However, as primary care physicians already use laboratory tests to help in making diagnoses, they may be more likely to include natriuretic peptides into their normal diagnostic repertoire. It remains to be seen, however, whether primary care physicians will modify therapy based on these results. It is also unknown whether this will lead eventually to physicians prescribing appropriate cardiac medications that are not part of their daily routine and ensuring that referrals to a cardiologist are followed through.

Natriuretic peptide assessment is not a stand alone test in the setting of distinguishing cardiac from pulmonary dyspneas; however, it does appear to be able to lead the physician in the right direction and unveil underlying cardiac issues. The study by Wieshammer et al. [6] helps to show the value of natriuretic peptides in this setting. Although natriuretic peptide assays are now common in the emergency departments of many hospitals, they are still foreign in primary care settings. Currently, we benefit from having this test available for acutely ill patients, and in the future we will hopefully be able to rely on it as an early marker for dyspnea of cardiac origin presenting in nonemergency settings.

\section{References}

1 Mueller C, Scholer A, Laule-Kilian K, et al: Use of B-type natriuretic peptide in the evaluation and management of acute dyspnea. $\mathrm{N}$ Engl J Med 2004;350:647-654.

-2 Mueller C, Breidthardt T, Laule-Kilian K, et al: The integration of BNP and NT-proBNP into clinical medicine. Swiss Med Wkly 2007; 137:4-12.

3 Peacock WF, Mueller C, Disomma S, Maisel A: Emergency department perspectives on B-type natriuretic peptide utility. Congest Heart Fail 2008;14(suppl 1):17-20.
-4 Rutten JH, Steyerberg EW, Boomsma F, et al: $\mathrm{N}$-terminal pro-brain natriuretic peptide testing in the emergency department: beneficial effects on hospitalization, costs, and outcome. Am Heart J 2008;156:71-77.

5 Daniels LB, Maisel AS: Natriuretic peptides. J Am Coll Cardiol 2007;50:2357-2368.

6 Wieshammer S, Dreyhaupt J, Basler B, Marsovszky E: NT-proBNP for pulmonologists: not only a rule-out test for systolic heart failure but also a global marker of heart disease. Respiration 2009;77:370-380.
7 Gegenhuber A, Mueller T, Dieplinger B, et al: B-type natriuretic peptide and amino terminal proBNP predict one-year mortality in short of breath patients independently of the baseline diagnosis of acute destabilized heart failure. Clin Chim Acta 2006;370:174-179.

-8 Maisel A, Mueller C, Adams K Jr, et al: State of the art: using natriuretic peptide levels in clinical practice. Eur J Heart Fail 2008; 10: 824-839.

9 Mueller C., et al: B-type natriuretic peptides for the evaluation of exercise intolerance. Am J Med 2009, in press. 